\title{
Transcatheter or surgical treatment of severe aortic stenosis and coronary artery disease: A comparative analysis from the Italian OBSERVANT study
}

\author{
Marco Barbanti a,*,1, Sergio Buccheri ${ }^{\mathrm{a}, 1}$, Davide Capodanno a , Paola D'Errigo ${ }^{\mathrm{b}}$, Marco Ranucci ${ }^{\mathrm{c}}$, \\ Stefano Rosato ${ }^{\mathrm{b}}$, Gennaro Santoro ${ }^{\mathrm{d}}$, Danilo Fusco ${ }^{\mathrm{e}}$, Corrado Tamburino ${ }^{\mathrm{a}}$, Fausto Biancari ${ }^{\mathrm{f}}$, \\ Fulvia Seccareccia ${ }^{\mathrm{b}}$, for the OBSERVANT Research Group \\ a Division of Cardiology, Ferrarotto Hospital, University of Catania, Italy \\ b National Centre for Global Health - Istituto Superiore di Sanità, Rome, Italy \\ c Department of Cardiothoracic and Vascular Anesthesia and ICU - IRCCS Policlinico San Donato, San Donato Milanese, Milan, Italy \\ ${ }^{\mathrm{d}}$ Division of Cardiology, Careggi Hospital, Florence, Italy \\ e Department of Epidemiology of Lazio Regional Health Service, Rome, Italy \\ ${ }^{\mathrm{f}}$ Department of Surgery, Oulu University Hospital, Oulu, Finland
}

\section{A R T I C L E I N F O}

\section{Article history:}

Received 7 November 2017

Received in revised form 14 February 2018

Accepted 4 June 2018

Available online 7 June 2018

\section{Keywords:}

Aortic stenosis

Coronary artery disease

TAVI

SAVR

PCI

CABG

\begin{abstract}
A B S T R A C T
Background: To assess clinical outcomes of patients with concomitant severe aortic stenosis (AS) and coronary artery disease (CAD) who underwent transcatheter aortic valve implantation (TAVI) and percutaneous coronary intervention (PCI) or surgical aortic valve replacement (SAVR) and coronary artery bypass grafting (CABG).

Methods: Data were extracted from the multicenter OBSERVANT study. For the purposes of the present analysis, we included only patients with established stable CAD meeting any of the following inclusion criteria: 1) TAVI patients with CAD undergoing staged PCI or TAVI and PCI in the same session; 2) SAVR patients undergoing combined SAVR and CABG in the same session.

Results: After propensity-score matching, a total of 472 patients (236 per group) were identified. Among TAVI patients, PCI was performed prior to the procedure in 217 patients (92.0\%), whereas concomitant TAVI and PCI were performed in 19 patients $(8.0 \%)$. At 3-year, there was no difference in survival between the two groups (KM estimate of freedom from death for SAVR and TAVI patients of 0.742 and 0.650 , respectively; $\log$-rank $p$ value of 0.105 ). The rate of MACCE was comparable between the two groups (KM estimate of freedom from MACCE for SAVR and TAVI patients of 0.683 and 0.582 , respectively; log-rank $p$-value of 0.115 ).

Conclusions: In patients with associated severe AS and CAD, percutaneous treatment (TAVR and staged or concomitant PCI) was comparable to surgical treatment (SAVR and concomitant CABG) with respect to the early and mid-term risk of death from any cause, myocardial infarction, stroke and unplanned revascularization.
\end{abstract}

(c) 2018 Elsevier B.V. All rights reserved.

\section{Introduction}

Coronary artery disease (CAD) is common in patients with severe aortic stenosis (AS), which does not surprise because these two pathologies share many causative factors [1]. The presence of concomitant CAD has been associated with adverse procedural outcomes in aortic valve replacement $[2,3]$.

\footnotetext{
* Corresponding author at: Division of Cardiology - Ferrarotto Hospital, University of Catania, Catania, Italy.

E-mail address: mbarbanti83@gmail.com (M. Barbanti).

1 Marco Barbanti and Sergio Buccheri contributed equally to this work.
}

If in surgical patients the current standard of care for patients with concomitant AS and CAD is to combine coronary artery bypass grafting (CABG) with surgical aortic valve replacement (SAVR) in the same session $[4,5]$, optimal management of CAD in the context of transcatheter aortic valve implantation (TAVI) is highly debated due to the lack of comprehensive and consistent data on this topic [6, 7]. However, in clinical practice, prophylactic percutaneous coronary intervention (PCI) or concomitant TAVI and PCI of coronaries supplying large myocardial areas are the most adopted approaches [7-10].

The comparative efficacy of TAVI and SAVR has been extensively investigated in large randomized trials [11-14] and propensity matchedbased observational studies [15]. However, patients with CAD requiring coronary revascularization were excluded from the majority of 
randomized trials or poorly investigated, thus creating an important gap in the current evidence [11-14].

To shed light on this controversial area, we report on the mid-term clinical outcomes of a large series of patients with concomitant severe AS and CAD who underwent TAVI and PCI or SAVR and CABG and were recruited in the Italian national multicenter OBSERVANT (Observational Study of Effectiveness of SAVR-TAVI Procedures for Severe Aortic Stenosis Treatment) study.

\section{Methods}

2.1. Study design and data quality assessment

OBSERVANT is a national observational, prospective, multicenter, cohort study that enrolled consecutive patients undergoing TAVI or SAVR for severe aortic valve stenosis at 93 Italian cardiology/cardiac surgery centers. Details on the study design, patient eligibility criteria, and data collection modalities of the OBSERVANT registry have been reported elsewhere $[16,17]$. This study was coordinated by the Italian National Institute of Health and led in cooperation with the Italian Ministry of Health, the National Agency for Regional Health Services, Italian Regions, and Italian scientific societies and federations representing Italian professionals involved in the management of severe AS. In the participating hospitals, both SAVR and TAVI were available options. Techniques and choice of the prosthesis were left to the operator's discretion according to local pre-interventional workout and institutional practice. The Ethical Committee of each participating center approved the study protocol, and patients gave their informed consent to participate in the study.

\subsection{Patient population}

Invasive coronary angiography was mandatory in all patients and was assessed by the local heart team. In case of significant CAD (i.e., $>50 \%$ diameter stenosis on visual assessment of the coronary angiogram), the treatment strategy and completeness of revascularization was determined based on consensus decision before TAVI or SAVR at each participating center. For the purposes of the present analysis, we included only patients with established stable CAD (i.e., documented by coronary angiography) meeting any of the following inclusion criteria: 1) TAVI patients with CAD undergoing PCI prior to the procedure ( $<6$ months before TAVI) or combined TAVI and PCI in the same session; 2) SAVR patients undergoing combined SAVR and CABG in the same session. Inoperable patients (i.e. porcelain aorta and hostile thorax) and patients undergoing hybrid $\mathrm{PCI}$ and $\mathrm{CABG}$ procedures, concomitant interventions in other valves, or those undergoing TAVI nontransfemoral access were excluded.

\subsection{Outcomes of interest}

The primary outcomes of interest were all-cause mortality and the composite of death, myocardial infarction, stroke and unplanned revascularization at 30 days, 1, 2 and 3 years from SAVR or TAVI. Unplanned revascularization was defined as any revascularization procedure (CABG or $\mathrm{PCI}$ ) performed at least 30 days after the index intervention or as revascularization for acute myocardial infarction at any time point.

\subsection{Follow up}

As part of the OBSERVANT study, an administrative follow-up has been set up for each enrolled patient through a record linkage with the National Hospital Discharged Records (HDR) database (for in-hospital events: re-hospitalization, stroke, acute myocardial infarction, $\mathrm{PCI}$ and $\mathrm{CABG}$ ) and with the Tax Registry Information System (TRIS) (for information on life status). Specific quality assessment activities were arranged to evaluate the reliability and coherence of the OBSERVANT database. In particular, independent observers, following specific standard operating procedures, monitored the participating hospitals to assess the completeness of the enrolled cohort and to compare the collected data to those reported in the original clinical charts.

\subsection{Statistical analysis}

Continuous variables are reported as mean and standard deviation (SD) while dichotomous parameters as frequencies and percentages (\%). The normal distribution of continuous parameters was tested with the Kolmogorov-Smirnov test. Variables with a skewed distribution were compared with the use of Wilcoxon rank sum tests. $t$-Test, Chi-square or Fisher exact tests were used to compare frequencies among groups, as appropriate. Unadjusted event rates at follow-up were plotted according to the Kaplan-Meier method and differences in survival were tested with the log-rank test. We used the cumulative incidence function to account for the competing risk of death with other events of interest (e.g. MI, stroke and unplanned revascularization). We then compared the cumulative incidence functions between SAVR and TAVI groups using the Gray test.

\subsection{Propensity score matching}

To account for the non-randomized design of our study, a propensity score has been estimated using a logistic regression model according to a non-parsimonious approach [18]. The following clinical pre-procedural variables were included in the model: age, gender, chronic obstructive pulmonary disease, diabetes, history of myocardial infarction, left ventricular ejection fraction, neurological disease, creatinine and hemoglobin levels, dialysis, Euroscore II-estimated risk of 30-day mortality, frailty, New York Heart Association functional class III or IV at presentation, moderate-to-severe mitral regurgitation, peripheral artery disease, mean gradient, pulmonary hypertension. Pairs of SAVR and TAVI patients having the same probability score (nearest neighbor method; caliper $=0.25 *$ SD $(\operatorname{logitPs}))$ have been matched with a 1:1 ratio. Standardized mean differences before and after matching were calculated and a standardized difference below $10 \%$ was considered as a criterion of balance between the study cohorts. In addition to weighting, a simultaneous multivariate adjustment (doubly robust estimate) was performed for covariates included in the propensity score model with an absolute standardized difference $>10 \%$ after weighting. Finally, predicted probabilities of survival from the adjusted Cox-model were obtained and plotted for the principal outcomes of interest.

All tests performed in the current analysis are two-tailed and a $p$-value $<0.05$ has been considered statistically significant. All statistical analyses were conducted in $\mathrm{R}$ statistical software (version 3.2.1) equipped with the "twang" and "survival" packages.

\section{Results}

A total of 7618 consecutive patients with severe AS were enrolled in the OBSERVANT study between December 2010 and June 2012. All patients underwent either SAVR $(n=5707)$ or TAVI $(n=1911)$ between December 2010 and June 2012. From this unselected cohort, a total of 1719 patients (1420 SAVR and 299 TAVI patients) met the inclusion/exclusion criteria for this post-hoc analysis and were included in the study. Administrative linkage was carried out in $100 \%$ of patients and follow-up was complete in all patients.

Clinical characteristics between the SAVR and TAVI groups are shown in Table 1. As expected, before matching there was a marked unbalance in covariates between the two groups.

\subsection{Propensity scores balance}

After matching, a total of 236 pairs of patients were identified. Differences between TAVI and SAVR patients were well corrected for most of covariates, except for creatinine, dialysis and low LVEF (<30\%), with standardized differences slightly above $10 \%$ (12.6, 10.8 and $11.6 \%$, respectively). To take into account this imbalance, all the outcome estimate provided below have been adjusted for these three covariate following the doubly robust estimate approach.

\subsection{Procedural characteristics of the matched cohorts}

All TAVI procedures were performed using the third-generation, selfexpanding CoreValve prosthesis (Medtronic Inc., Galway, Ireland) or the balloon-expandable Edwards SAPIEN XT (Edwards Lifescience, Irvine, CA). Among TAVI patients, $\mathrm{PCI}$ was performed prior to the procedure in 217 patients (92.0\%), whereas concomitant TAVI and $\mathrm{PCI}$ were performed in 19 patients (8.0\%). All SAVR patients underwent concomitant valve replacement and CABG.

\subsection{Periprocedural and in-hospital outcomes}

Moderate or severe paravalvular regurgitation (0.6\% vs. $14.3 \%, p<$ $0.001)$, vascular complications ( $0.8 \%$ vs. $9.3 \%, p<0.001)$ and high degree conduction disturbances requiring pacemaker implantation (3.0\% vs. $17.4 \%, p<0.001$ ) were more frequently encountered in TAVI patients. Conversely, acute kidney injury (14.0\% vs. $2.5 \%, p \leq 0.001)$ and bleeding requiring $>4$ units of $\mathrm{RBCs}(15.3 \%$ vs. $3.4 \%, p<0.001)$ were more frequently reported in the surgical cohort. 
Table 1

Clinical characteristics between the two groups.

\begin{tabular}{|c|c|c|c|c|c|c|}
\hline & \multicolumn{2}{|c|}{ Before matching } & \multicolumn{2}{|c|}{ After matching } & \multicolumn{2}{|l|}{ SMD (\%) } \\
\hline & SAVR & TAVI & SAVR & TAVI & Before matching & After matching \\
\hline $\mathrm{n}$ & 1420 & 299 & 236 & 236 & & \\
\hline Age, mean (SD) & $75.14(7.5)$ & $81.66(6.0)$ & $80.5(5.2)$ & $80.7(6.2)$ & 95.8 & 3.6 \\
\hline Euroscore II, mean (SD) & $5.5(5.5)$ & $8.2(8.7)$ & $7.8(6.0)$ & $7.8(8.8)$ & 36.2 & 0.6 \\
\hline Male gender, n (\%) & $900(63.4)$ & $129(43.1)$ & $132(55.9)$ & $128(54.2)$ & 41.4 & 3.4 \\
\hline Diabetes, n (\%) & $466(32.8)$ & $98(32.8)$ & $80(33.9)$ & $77(32.6)$ & 0.1 & 2.7 \\
\hline Prior MI, n (\%) & $319(22.5)$ & $86(28.8)$ & $62(26.3)$ & $68(28.8)$ & 14.5 & 5.7 \\
\hline BMI, mean (SD) & $27.0(4.2)$ & $25.9(4.7)$ & $26.1(4.1)$ & $26.0(4.7)$ & 25.4 & 2.5 \\
\hline Neurological disorder, $\mathrm{n}(\%)$ & $41(2.9)$ & $30(10.0)$ & $19(8.1)$ & $21(8.9)$ & 29.4 & 3.0 \\
\hline COPD, n (\%) & $156(11.0)$ & $79(26.4)$ & $56(23.7)$ & $53(22.5)$ & 40.4 & 3.0 \\
\hline Creatinine, mean (SD) & $1.19(1.06)$ & $1.29(0.95)$ & $1.16(0.85)$ & $1.27(1.00)$ & 9.9 & 12.6 \\
\hline Frailty moderate or severe ${ }^{a}, \mathrm{n}(\%)$ & $91(6.4)$ & $73(24.4)$ & $45(19.1)$ & $43(18.2)$ & 51.5 & 2.2 \\
\hline Hemoglobin, mean (SD) & $12.4(1.8)$ & $11.5(1.6)$ & $11.7(1.8)$ & $11.6(1.6)$ & 56.3 & 7.3 \\
\hline NYHA class III/IV, n (\%) & $566(39.9)$ & $186(62.2)$ & $139(58.9)$ & $132(55.9)$ & 45.9 & 6.0 \\
\hline $\mathrm{PAD}, \mathrm{n}(\%)$ & $328(23.1)$ & $87(29.1)$ & $67(28.4)$ & $69(29.2)$ & 13.7 & 1.9 \\
\hline Dialysis, n (\%) & $37(2.6)$ & $8(2.7)$ & $4(1.7)$ & $8(3.4)$ & 0.4 & 10.8 \\
\hline LVEF < $30 \%, n(\%)$ & $30(2.1)$ & $14(4.7)$ & $15(6.4)$ & $9(3.8)$ & 14.2 & 11.6 \\
\hline Mean gradient ( $\mathrm{mm} \mathrm{Hg})$, mean (SD) & $47.5(15.3)$ & $47.5(13.3)$ & & & & \\
\hline MR moderate-severe, $\mathrm{n}(\%)$ & $184(13.0)$ & $83(27.8)$ & $59(25.0)$ & $53(22.5)$ & 37.4 & 6.0 \\
\hline $\mathrm{sPAP}>60 \mathrm{~mm} \mathrm{Hg}, \mathrm{n}(\%)$ & $77(5.4)$ & $45(15.1)$ & $29(12.3)$ & $28(11.9)$ & 32.2 & 1.3 \\
\hline
\end{tabular}

Abbreviations: SMD, standardized mean difference; KS, Kolmogorov-Smirnov; MI, myocardial infarction; MR, mitral regurgitation; BMI, body mass index; EF, ejection fraction; COPD, chronic obstructive pulmonary disease; NYHA, New York Heart Association; PAD, peripheral artery disease; sPAP, systolic pulmonary arterial pressure

a Defined according to Geriatric Status Scale.

\subsection{Three-year clinical outcomes}

Survival curves for SAVR and TAVI matched cohorts are shown in Fig. 1. At 3-year, there was no difference in survival between the two groups (KM estimate of freedom from death for SAVR and TAVI patients of 0.742 and 0.650 , respectively; log-rank $p$-value of 0.105$)$. Similarly, the rate of MACCE was comparable between the two groups (KM estimate of freedom from MACCE for SAVR and TAVI patients of 0.683 and 0.582 , respectively; log-rank p-value of 0.115). The cumulative number of events for MACCE and death at different follow-up intervals is reported in Table 2. The cumulative incidence of MI, stroke and revascularization is shown in Table 3. There were no differences between the two groups for all these endpoints at 3-year follow-up (all $p$-values by Gray test $>0.05$ ).

\section{Discussion}

The principal finding of this study including a population of patients with associated severe AS and CAD was that percutaneous treatment (TAVI and PCI prior to or combined during the procedure) was comparable to surgical treatment (SAVR and concomitant CABG) with respect to the 30-day, 1 year and 3-year rates of death from any cause, stroke, myocardial infarction and unplanned revascularization. However, at 3 years, a trend toward lower survival from death and from the composite of death, MI, stroke and unplanned revascularization was seen in patients treated with TAVI.

Despite the availability of several head-to-head comparisons between TAVI and SAVR in randomized clinical trials and meta-analyses of elderly patients at high and intermediate surgical risk [11-15], it is still unknown which approach performs better when severe AS and CAD coexist in this particular population. In fact, concomitant CAD requiring treatment was an exclusion criterion of the majority of the most representative trials comparing SAVR and TAVI (PARTNER 1, CoreValve U.S. and NOTION) [11-13], and was not deeply investigated in the PARTNER 2 trial. However, CAD patients represent a sizeable proportion of subjects referred to TAVI or SAVR in real-world practice. CAD is frequently seen in patients with severe AS $[1,7]$. Therefore, understanding which approach should be preferred in patients with both pathologies would have a great impact, particularly in the current era, in which TAVI is being increasingly used to treat younger and lower risk patients with a longer life expectancy than those who underwent TAVI in the past. This analysis of the OBSERVANT study aims to fill this
Survival from all-cause death

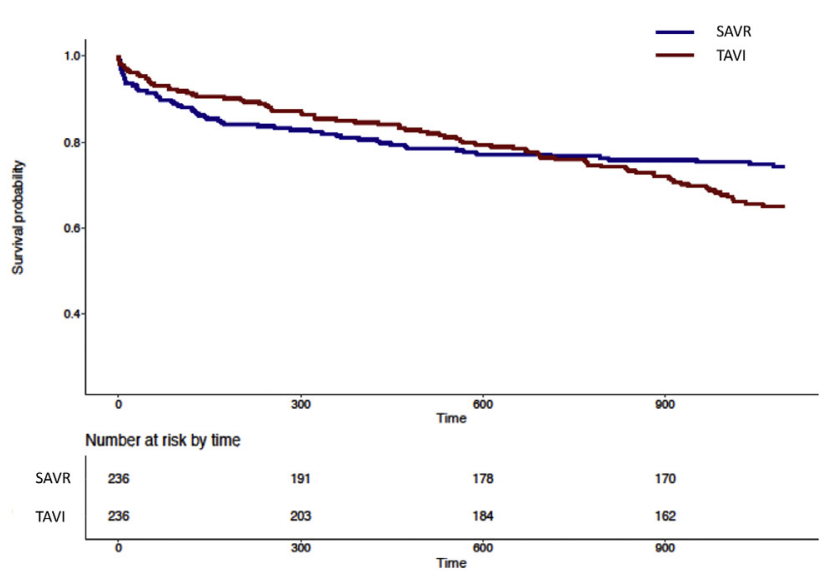

Survival from MACCE

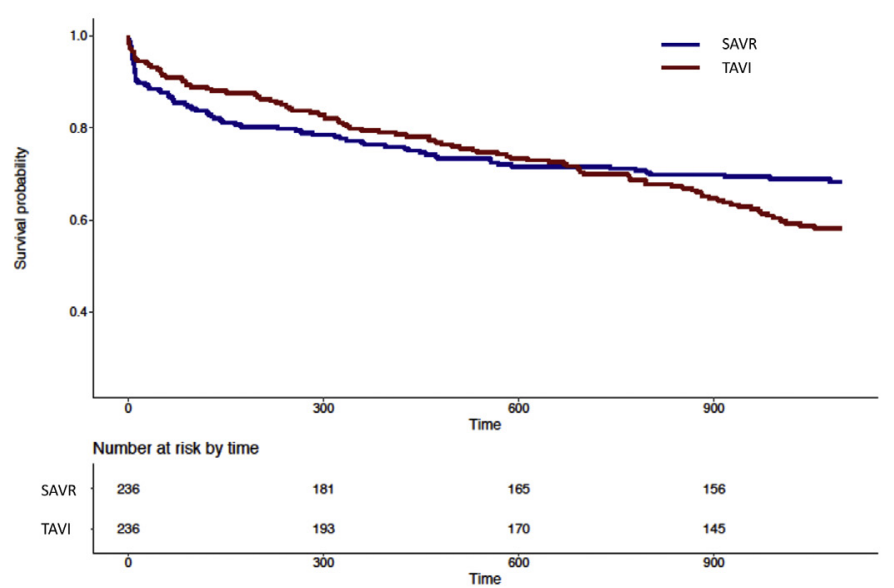

Fig. 1. Kaplan-Meier curves illustrating survivals free from all-cause death and MACCE (death, stroke, MI and unplanned revascularization). 
Table 2

Clinical outcomes at different follow-up intervals in matched cohorts

\begin{tabular}{|c|c|c|c|c|c|}
\hline \multirow[t]{2}{*}{ Outcome of interest } & \multirow[t]{2}{*}{ Timing } & \multicolumn{2}{|c|}{ Cumulative events } & \multicolumn{2}{|l|}{ KM estimate (95\%CI) } \\
\hline & & SAVR & TAVI & SAVR & TAVI \\
\hline \multirow[t]{4}{*}{ Death } & 30-day & 18 & 9 & $0.923(0.890-0.958)$ & $0.962(0.937-0.987)$ \\
\hline & 1 year & 43 & 35 & $0.815(0.767-0.867)$ & $0.850(0.805-0.897)$ \\
\hline & 2 year & 54 & 56 & $0.767(0.715-0.824)$ & $0.759(0.707-0.816)$ \\
\hline & 3 year & 59 & 79 & $0.742(0.687-0.801)^{*}$ & $0.650(0.590-0.716)^{*}$ \\
\hline \multirow[t]{4}{*}{ MACCE } & 30-day & 26 & 14 & $0.890(0.850-0.930)$ & $0.940(0.911-0.971)$ \\
\hline & 1 year & 55 & 48 & $0.764(0.711-0.820)$ & $0.794(0.744-0.848)$ \\
\hline & 2 year & 66 & 70 & $0.716(0.660-0.776)$ & $0.700(0.643-0.761)$ \\
\hline & 3 year & 73 & 95 & $0.683(0.625-0.746)^{*}$ & $0.582(0.520-0.650)^{*}$ \\
\hline
\end{tabular}

Abbreviations: KM, Kaplan Meier; TAVI, transcatheter aortic valve implantation; SAVR, surgical aortic valve replacement; MI, myocardial infarction

* Log-rank $p$-values for 3-year outcomes: death 0.105; MACCE 0.115.

important evidence gap, until properly designed randomized trials will be available.

According to the most recent North American and European guidelines, SAVR patients with significant CAD should undergo concomitant CABG $[4,5]$, as it was demonstrated that this approach reduces both short-term mortality and increases long-term survival up to 10 years [19]. On the contrary, optimal management of CAD in the context of TAVI is highly debated [7]. There is only a general consensus that severe coronary lesions that subtend a large area of myocardium such as proximal epicardial lesions should be considered for PCI before or eventually at the time of TAVI [7]. According to these different treatment strategies, it is reasonably assumed that complete revascularization is considerably more often obtained in surgical patients as compared with those undergoing TAVI and PCI $[7,9,10]$. Whether these two strategies lead different outcomes in elderly and intermediate or high-risk patients remains to be determined. Indeed, this study represents the first attempt to shed more light on this topic.

In our study, we demonstrated that the death from any cause and MACCE rates up to 3 years were similar in the percutaneous and surgical groups, even though it must be pointed out that at 3 years, there was a trend toward increased mortality and cumulative MACCE rates in TAVI patients. We did not observe significant differences in terms of myocardial infarction and stroke. A potential contributing explanation for this finding might be a lower rate of complete revascularization obtained in TAVI patients with PCI as compared with CABG. Indeed, CAD left untreated may be the substrate for future cardiovascular events leading to a marginal increase in the risk of mortality at long-term follow-up. It has to be acknowledged that the actual need for complete revascularization in TAVI patients is poorly investigated and that the extent of residual CAD left untreated may differ based on age and individual risk characteristics [20, 21]. Indeed, Girerd et al. found that incomplete revascularization did not have an impact on survival in patients $>60$ years of age, suggesting that in this particular elderly patient population at high

Table 3

Cumulative incidence functions at different follow-up intervals in matched cohorts.

\begin{tabular}{|c|c|c|c|c|c|}
\hline \multirow[t]{2}{*}{ Outcome of interest } & \multirow[t]{2}{*}{ Timing } & \multicolumn{2}{|c|}{$\begin{array}{l}\text { Cumulative } \\
\text { events }\end{array}$} & \multicolumn{2}{|c|}{$\begin{array}{l}\text { Cumulative } \\
\text { incidence function }\end{array}$} \\
\hline & & SAVR & TAVI & SAVR & TAVI \\
\hline \multirow[t]{4}{*}{ MI } & 30-day & 9 & 5 & $1.28 \%$ & $1.27 \%$ \\
\hline & 1 year & 10 & 11 & $2.15 \%$ & $3.85 \%$ \\
\hline & 2 year & 11 & 14 & $2.58 \%$ & $4.71 \%$ \\
\hline & 3 year & 14 & 19 & $3.49 \% *$ & $6.53 \%^{*}$ \\
\hline \multirow[t]{4}{*}{ Stroke } & 30-day & 3 & 3 & $1.27 \%$ & $1.27 \%$ \\
\hline & 1 year & 7 & 14 & $3.00 \%$ & $6.00 \%$ \\
\hline & 2 year & 9 & 15 & $3.87 \%$ & $6.43 \%$ \\
\hline & 3 year & 13 & 17 & $5.67 \% *$ & $7.30 \% *$ \\
\hline \multirow[t]{4}{*}{ Revascularization } & 30-day & 0 & 1 & $1.27 \%$ & $3.39 \%$ \\
\hline & 1 year & 1 & 5 & $1.27 \%$ & $3.39 \%$ \\
\hline & 2 year & 2 & 7 & $1.27 \%$ & $3.39 \%$ \\
\hline & 3 year & 3 & 8 & $1.27 \%^{*}$ & $3.39 \%^{*}$ \\
\hline
\end{tabular}

* $p$-Values by Gray test of 0.444 for MI, 0.966 for stroke and 0.128 for revascularization. operative risk, pursuing complete revascularization is not mandatory, provided that a rational approach to CAD by a dedicated heart team is guaranteed [20]. More recent studies on TAVI patients also confirmed this observation, showing that as long as the target population is old and at high-risk, a judicious revascularization strategy selection is associated with favorable mid-term outcomes, obviating the need for complete CAD revascularization $[9,10]$. The concept of reasonable extent of residual CAD left untreated after PCI or CABG has been the object of numerous investigations [22,23], but whether this also applies to the TAVI scenario is unknown and deserves future investigations. The actual need for PCI compared with medical therapy in TAVI patients is the object of an ongoing trial (ISRCTN75836930) [24].

Finally, it should be acknowledged that among the OBSERVANT study, TAVI patients were treated with previous-generation devices; technical developments with newly generation of percutaneous valves are already showing to reduce a procedural gap in comparison to SAVR in patients with isolated AS [25, 26].

\subsection{Study limitations}

This study has different limitations. First, it is not a randomized trial. Although we conducted an extensive statistical adjustment (including a 1:1 propensity-score matching, presented in the Supplementary appendix), the impact of unidentified confounders is an unavoidable limitation of observational studies. However, it has been argued that a wellconducted observational cohort study can provide the same level of internal validity as randomized controlled trials. Moreover, observational studies are carried out on real-world populations, and therefore can reach higher levels of external validity compared to RCTs. Second, the OBSERVANT study did not collect extensive details regarding coronary revascularization strategies, including number and type of stent and grafts and PCI/CABG target vessels, or information on completeness of revascularization. Third, data on noninvasive functional assessments of ischemia using nuclear perfusion imaging, echocardiography, or magnetic resonance imaging were not collected in the database. Finally, another limitation of the present study is that the outcome events were not defined according to Valve Academic Research Consortium (VARC). The reason is that such definitions are specifically designed to define complications after TAVR and therefore may be misleading to illustrate complications after SAVR, likely resulting in their overestimation. Furthermore, the OBSERVANT study was started on before these guidelines were published.

\section{Conclusions}

In patients with associated severe AS and CAD a fully percutaneous treatment (TAVR and staged or concomitant PCI) was comparable to surgical treatment (SAVR and concomitant CABG) with respect to the early and mid-term risk of death from any cause, myocardial infarction, stroke and unplanned revascularization. Further studies with newer 
generation TAVI devices and longer term follow-up are needed to confirm our findings.

\section{Disclosure}

Dr. Barbanti is consultant for Edwards Lifesciences. Prof. Tamburino is consultant for Medtronic and St Jude Medical. All other authors have no disclosure.

\section{Appendix A. Supplementary data}

Supplementary data to this article can be found online at https://doi. org/10.1016/j.ijcard.2018.06.011.

\section{References}

[1] B.F. Stewart, D. Siscovick, B.K. Lind, et al., Clinical factors associated with calcific aortic valve disease. Cardiovascular Health Study, J. Am. Coll. Cardiol. 29 (1997) 630-634.

[2] Y.S. Tjang, Y. van Hees, R. Korfer, et al., Predictors of mortality after aortic valve replacement, Eur. J. Cardiothorac. Surg. 32 (2007) 469-474.

[3] D.S. Likosky, M.J. Sorensen, L.J. Dacey, et al., Long-term survival of the very elderly undergoing aortic valve surgery, Circulation 120 (2009) S127-S133.

[4] H. Baumgartner, V. Falk, J.J. Bax, et al., 2017 ESC/EACTS Guidelines for the management of valvular heart disease, Eur. Heart J. 38 (2017) 2739-2791.

[5] R.A. Nishimura, C.M. Otto, R.O. Bonow, et al., 2017 AHA/ACC focused update of the $2014 \mathrm{AHA} / \mathrm{ACC}$ guideline for the management of patients with valvular heart disease: a report of the American College of Cardiology/American Heart Association Task Force on Clinical Practice Guidelines, J. Am. Coll. Cardiol. 70 (2017) 252-289.

[6] F. D'Ascenzo, F. Conrotto, F. Giordana, et al., Mid-term prognostic value of coronary artery disease in patients undergoing transcatheter aortic valve implantation: a metaanalysis of adjusted observational results, Int. J. Cardiol. 168 (2013) 2528-2532.

[7] S.S. Goel, M. Ige, E.M. Tuzcu, et al., Severe aortic stenosis and coronary artery diseaseimplications for management in the transcatheter aortic valve replacement era: a comprehensive review, J. Am. Coll. Cardiol. 62 (2013) 1-10.

[8] P. Wenaweser, T. Pilgrim, E. Guerios, et al., Impact of coronary artery disease and percutaneous coronary intervention on outcomes in patients with severe aortic stenosis undergoing transcatheter aortic valve implantation, EuroIntervention 7 (2011) 541-548.

9] G.P. Ussia, M. Barbanti, A. Colombo, et al., Impact of coronary artery disease in elderly patients undergoing transcatheter aortic valve implantation: insight from the Italian CoreValve Registry, Int. J. Cardiol. 167 (2013) 943-950.

[10] M. Barbanti, D. Todaro, G. Costa, et al., Optimized screening of coronary artery disease with invasive coronary angiography and ad hoc percutaneous coronary intervention during transcatheter aortic valve replacement, Circ. Cardiovasc. Interv. 10 (8) (2017 Aug), https://doi.org/10.1161/CIRCINTERVENTIONS.117.005234.
[11] C.R. Smith, M.B. Leon, M.J. Mack, et al., Transcatheter versus surgical aortic-valve replacement in high-risk patients, N. Engl. J. Med. 364 (2011) 2187-2198.

[12] D.H. Adams, J.J. Popma, M.J. Reardon, et al., Transcatheter aortic-valve replacement with a self-expanding prosthesis, N. Engl. J. Med. 370 (2014) 1790-1798.

[13] H.G. Thyregod, D.A. Steinbrüchel, N. Ihlemann, et al., Transcatheter versus surgical aortic valve replacement in patients with severe aortic valve stenosis: 1 -year results from the All-comers NOTION randomized clinical trial, J. Am. Coll. Cardiol. 65 (2015) 2184-2194.

[14] M.B. Leon, C.R. Smith, M.J. Mack, et al., Transcatheter or surgical aortic-valve replacement in intermediate-risk patients, N. Engl. J. Med. 374 (2016) 1609-1620.

[15] G. Gargiulo, A. Sannino, D. Capodanno, et al., Transcatheter aortic valve implantation versus surgical aortic valve replacement: a systematic review and meta-analysis, Ann. Intern. Med. 165 (5) (2016 Sep 6) 334-344.

[16] P. D'Errigo, M. Barbanti, M. Ranucci, et al., Transcatheter aortic valve implantation versus surgical aortic valve replacement for severe aortic stenosis: results from an intermediate risk propensity-matched population of the Italian OBSERVANT study, Int. J. Cardiol. 167 (2013) 1945-1952.

[17] C. Tamburino, M. Barbanti, P. D'Errigo, et al., 1-year outcomes after transfemoral transcatheter or surgical aortic valve replacement: results from the Italian OBSERVANT study, J. Am. Coll. Cardiol. 66 (2015) 804-812.

[18] D.F. Mccaffrey, B.A. Griffin, D. Almirall, et al., A tutorial on propensity score estimation for multiple treatments using generalized boosted models, Stat. Med. 32 (2013) $3388-3414$.

[19] O. Lund, T.T. Nielsen, H.K. Pilegaard, et al., The influence of coronary artery disease and bypass grafting on early and late survival after valve replacement for aortic stenosis, J. Thorac. Cardiovasc. Surg. 100 (1990) 327-337.

[20] N. Girerd, J. Magne, M. Rabilloud, et al., The impact of complete revascularization on long-term survival is strongly dependent on age, Ann. Thorac. Surg. 94 (2012) $1166-1172$.

[21] A. Maslow, P. Casey, A. Poppas, et al., Aortic valve replacement with or without coronary artery bypass graft surgery: the risk of surgery in patients $>$ or $=80$ years old, J. Cardiothorac. Vasc. Anesth. 24 (2010) 18-24.

[22] V. Farooq, C. Girasis, M. Magro, et al., The CABG SYNTAX Score - an angiographic tool to grade the complexity of coronary disease following coronary artery bypass graft surgery: from the SYNTAX Left Main Angiographic (SYNTAX-LE MANS) substudy, EuroIntervention 8 (2013) 1277-1285.

[23] V. Farooq, P.W. Serruys, C.V. Bourantas, et al., Quantification of incomplete revascularization and its association with five-year mortality in the synergy between percutaneous coronary intervention with taxus and cardiac surgery (SYNTAX) trial validation of the residual SYNTAX score, Circulation 128 (2013) 141-151.

[24] M.Z. Khawaja, D. Wang, S. Pocock, et al., The percutaneous coronary intervention prior to transcatheter aortic valve implantation (ACTIVATION) trial: study protocol for a randomized controlled trial, Trials 15 (2014) 300.

[25] M. Barbanti, J.G. Webb, M. Gilard, et al, Transcatheter aortic valve implantation in 2017: state of the art, Eurolntervention 13 (2017) AA11-AA21.

[26] M. Barbanti, S. Buccheri, J. Rodés-Cabau, et al., Transcatheter aortic valve replacement with new-generation devices: a systematic review and meta-analysis, Int. J. Cardiol. 245 (2017) 83-89. 


\section{SUPPLEMENTARY APPENDIX}

Transcatheter or Surgical Treatment of Severe Aortic Stenosis and Coronary Artery Disease: A comparative analysis from the Italian OBSERVANT Study

Running title: Treatment options for severe aortic stenosis and coronary artery disease

Marco Barbanti, MD*, Sergio Buccheri, MD*, Davide Capodanno, MD*, Paola D’Errigo§, Marco

Ranucci, MD $\ddagger$, Stefano Rosato§, Gennaro Santoro, MD\|, Danilo Fusco $\uparrow \dagger$, Corrado Tamburino, MD*, Fausto Biancari, MD, PhD ๆ and Fulvia Seccareccia $\S$ for the OBSERVANT Research Group

*Division of Cardiology, Ferrarotto Hospital, University of Catania, Italy; §National Centre for Epidemiology, Surveillance and Health Promotion - Istituto Superiore di Sanità, Rome, Italy; †Department of Cardiothoracic and Vascular Anesthesia and ICU - IRCCS Policlinico San Donato, San Donato Milanese (Milan), Italy; ||Division of Cardiology, Careggi Hospital, Florence, Italy; $\dagger \dagger$ Department of Epidemiology of Lazio Regional Health Service, Rome, Italy; $\uparrow$ Department of Surgery, Oulu University Hospital, Oulu, Finland; Marco Barbanti and Sergio Buccheri contributed equally to this work.

Correspondence: Marco Barbanti M.D.

Division of Cardiology - Ferrarotto Hospital, University of Catania, Catania, ITALY.

Phone +39 095743 6103; Fax +39 095743 6105, e-mail: mbarbanti83@gmail.com 


\section{Methods}

Propensity score weighting - We used propensity score weighting to confirm the results of propensity matched analyses and avoid loss of information from unmatched patients and results are presented in the present Supplementary appendix. To obtain weights, we used an alternative methodology based on generalized boosted regression. The same covariates used for conventional propensity score calculation were included in this model. Generalized boosted regression algorithms estimate propensity score through an iterative process based on the analysis of multiple regression trees. Results of the sequential and multiple partitioning of the dataset are then used collectively to estimate the propensity score. The use of this method has several advantages, including the possibility 1) to analyze a large number of covariates without concern for model overfitting, handling complex and non-linear relationships between baseline covariates and treatment assignment variables, and 2) the opportunity to refine the balance of covariates using proper tuning parameters. [18] To estimate the propensity score in our dataset, we used 100,000 iterations and a shrinkage parameter of 0.001 . The iteration-stopping rule was based on the minimization of the Kolgomorov-Smirnov (KS) statistics mean. The balance of the propensity score was evaluated by plotting the absolute standardized difference before and after weighting. Moreover, the balance of covariates was evaluated with a Q-Q plot comparing the quantiles of the observed KolgomorovSmirnov statistic p-values before and after weighting. Propensity score estimates for each patient were finally used to obtain proportional weights [average treatment effects on the treated weights] that were entered as a weighting factor in Cox adjusted analyses. Average treatment effects on the treated weights were set at 1 for treated patients (TAVI group) and calculated as propensity score/(1-propensity score) for control subjects (SAVR group). The relationship between treatment assignment and the principal outcomes of interest was evaluated with a weighted Cox proportional hazard model. In addition to weighting, a simultaneous multivariate adjustment (doubly robust estimate) was performed for covariates included in the propensity score model with an absolute standardized difference $>0.1$ and $<0.2$ after weighting. Finally, predicted probabilities of survival 
from the adjusted Cox-model were obtained and plotted for the principal outcomes of interest in each group. 
eTable 1 Clinical characteristics between the two groups. The propensity score weighting was effective at reducing the unbalance in baseline characteristics between the TAVI and SAVR groups. A significant increase in Kolgomorov-Smirnov statistic p-values was observed after weighting and all weighted p-values were above the $45^{\circ}$ degree reference line.

\begin{tabular}{|c|c|c|c|c|c|c|}
\hline & \multicolumn{4}{|c|}{ Before weighting } & \multicolumn{2}{|c|}{ After weighting } \\
\hline & $\begin{array}{c}\text { SAVR } \\
(\mathrm{n}=1420)\end{array}$ & $\begin{array}{c}\text { TAVI } \\
(\mathrm{n}=299)\end{array}$ & SMD & $\begin{array}{l}\text { KS p- } \\
\text { value }\end{array}$ & SMD & $\begin{array}{l}\text { KS p- } \\
\text { value }\end{array}$ \\
\hline Age, mean (sd) & $75.14(7.5)$ & $81.66(6.0)$ & $108.7 \%$ & $<0.001$ & $19.8 \%$ & 0.165 \\
\hline Euroscore II, mean (sd) & $5.5(5.5)$ & $8.16(8.7)$ & $30.3 \%$ & $<0.001$ & $4.1 \%$ & 0.526 \\
\hline Male gender, $\mathrm{n}(\%)$ & $900(63.4)$ & $129(43.1)$ & $40.8 \%$ & $<0.001$ & $13.8 \%$ & 0.389 \\
\hline Diabetes, $\mathrm{n}(\%)$ & $466(32.8)$ & $98(32.8)$ & $0.1 \%$ & 1.00 & $3.2 \%$ & 1.00 \\
\hline Prior MI, n (\%) & $319(22.5)$ & $86(28.8)$ & $13.9 \%$ & 0.268 & $5.1 \%$ & 1.00 \\
\hline BMI, mean (sd) & $27.0(4.2)$ & $25.9(4.7)$ & $24.0 \%$ & $<0.001$ & $2.6 \%$ & 0.502 \\
\hline Neurological disorder, $\mathrm{n}(\%)$ & $41(2.9)$ & $30(10.0)$ & $23.7 \%$ & 0.152 & $12.0 \%$ & 0.973 \\
\hline COPD, n $(\%)$ & $156(11.0)$ & $79(26.4)$ & $34.9 \%$ & $<0.001$ & $12.5 \%$ & 0.656 \\
\hline Creatinine, mean (sd) & $1.19(1.1)$ & $1.29(0.9)$ & $10.5 \%$ & 0.002 & $2.1 \%$ & 0.821 \\
\hline Frailty moderate or severe*, n (\%) & $91(6.4)$ & $73(24.4)$ & $41.8 \%$ & $<0.001$ & $9.9 \%$ & 0.904 \\
\hline Hemoglobin, mean (sd) & $12.4(1.8)$ & $11.5(1.6)$ & $59.3 \%$ & $<0.001$ & $10.9 \%$ & 0.736 \\
\hline NYHA class III/IV, n (\%) & $566(39.9)$ & $186(62.2)$ & $46.0 \%$ & $<0.001$ & $13.7 \%$ & 0.424 \\
\hline PAD, n (\%) & $328(23.1)$ & $87(29.1)$ & $13.2 \%$ & 0.321 & $5.6 \%$ & 1.00 \\
\hline Dialysis, $\mathrm{n}(\%)$ & $37(2.6)$ & $8(2.7)$ & $0.4 \%$ & 1.000 & $3.6 \%$ & 1.00 \\
\hline $\mathrm{LVEF}<30 \%, \mathrm{n}(\%)$ & $30(2.1)$ & $14(4.7)$ & $12.1 \%$ & 0.995 & $8.3 \%$ & 1.00 \\
\hline Mean gradient $(\mathrm{mmHg})$, mean $(\mathrm{sd})$ & $47.5(15.3)$ & $47.5(13.3)$ & $2.1 \%$ & 0.988 & $1.8 \%$ & 1.00 \\
\hline MR moderate-severe, $\mathrm{n}(\%)$ & $184(13.0)$ & $83(27.8)$ & $33.0 \%$ & $<0.001$ & $13.9 \%$ & 0.506 \\
\hline $\mathrm{sPAP}>60 \mathrm{mmHg}, \mathrm{n}(\%)$ & $77(5.4)$ & $45(15.1)$ & $26.9 \%$ & 0.019 & $19.4 \%$ & 0.365 \\
\hline
\end{tabular}

Abbreviations: SMD, Standardized Mean Difference; KS, Kolgomorov-Smirnov; MI, myocardial infarction; MR, mitral regurgitation; BMI, body mass index; EF, ejection fraction; COPD, chronic obstructive pulmonary disease; NYHA, New York Heart Association; PAD, peripheral artery disease; sPAP, systolic Pulmonary Arterial Pressure. *Defined according to Geriatric Status Scale. 
eTable 2 Unadjusted and Cox-predicted survival estimates at different follow-up intervals of the entire cohort.

\begin{tabular}{|c|c|c|c|c|c|}
\hline $\begin{array}{l}\text { Outcome of } \\
\text { interest }\end{array}$ & Timing & $\begin{array}{c}\text { SAVR } \\
(\mathrm{n}=1420)\end{array}$ & $\begin{array}{c}\text { TAVI } \\
(\mathrm{n}=299)\end{array}$ & $\begin{array}{c}\text { SAVR } \\
(\mathrm{n}=1420)\end{array}$ & $\begin{array}{c}\text { TAVI } \\
(\mathrm{n}=299)\end{array}$ \\
\hline & & Unadjusted & Unadjusted & Adjusted Cox- & Adjusted Cox \\
\hline & & $\begin{array}{c}\text { KM estimate } \\
(95 \% \mathrm{CI})\end{array}$ & $\begin{array}{c}\text { KM estimate } \\
(95 \% \mathrm{CI})\end{array}$ & $\begin{array}{c}\text { Model predicted } \\
\text { survival }(95 \% \mathrm{CI})\end{array}$ & $\begin{array}{l}\text { Model predicted } \\
\text { survival }(95 \% \mathrm{CI})\end{array}$ \\
\hline \multirow[t]{4}{*}{ Death } & 30-day & $0.965(0.955-0.974)$ & $0.956(0.933-0.980)$ & $0.929(0.896-0.963)$ & $0.961(0.940-0.982)$ \\
\hline & 1 year & $0.892(0.876-0.909)$ & $0.835(0.793-0.878)$ & $0.829(0.780-0.880)$ & $0.846(0.806-0.888)$ \\
\hline & 2 year & $0.857(0.838-0.875)$ & $0.743(0.695-0.795)$ & $0.778(0.724-0.835)$ & $0.757(0.709-0.808)$ \\
\hline & 3 year & $0.829(0.809-0.849)$ & $0.641(0.587-0.700)$ & $0.749(0.692-0.810)$ & $0.657(0.602-0.716)$ \\
\hline \multirow[t]{4}{*}{ MI } & 30-day & $0.977(0.969-0.985)$ & $0.976(0.959-0.994)$ & $0.967(0.944-0.991)$ & $0.978(0.961-0.995)$ \\
\hline & 1 year & $0.969(0.959-0.978)$ & $0.953(0.928-0.978)$ & $0.963(0.938-0.989)$ & $0.956(0.932-0.981)$ \\
\hline & 2 year & $0.960(0.949-0.970)$ & $0.935(0.906-0.966)$ & $0.951(0.922-0.981)$ & $0.940(0.911-0.969)$ \\
\hline & 3 year & $0.953(0.941-0.964)$ & $0.908(0.872-0.947)$ & $0.946(0.915-0.978)$ & $0.914(0.878-0.952)$ \\
\hline \multirow[t]{4}{*}{ Stroke } & 30-day & $0.976(0.968-0.984)$ & $0.990(0.978-1.000)$ & $0.975(0.956-0.995)$ & $0.991(0.981-1.000)$ \\
\hline & 1 year & $0.965(0.955-0.974)$ & $0.948(0.922-0.975)$ & $0.961(0.935-0.987)$ & $0.955(0.930-0.981)$ \\
\hline & 2 year & $0.954(0.943-0.965)$ & $0.940(0.912-0.969)$ & $0.950(0.920-0.981)$ & $0.948(0.921-0.976)$ \\
\hline & 3 year & $0.941(0.928-0.954)$ & $0.931(0.901-0.962)$ & $0.943(0.910-0.977)$ & $0.940(0.910-0.971)$ \\
\hline \multirow[t]{4}{*}{ Revascularization } & 30-day & $0.999(0.998-1.000)$ & $1.000(1.000-1.000)$ & $1.000(0.997-1.000)$ & $1.000(1.000-1.000)$ \\
\hline & 1 year & $0.988(0.981-0.994)$ & $0.981(0.965-0.998)$ & $0.995(0.987-1.000)$ & $0.986(0.973-1.000)$ \\
\hline & 2 year & $0.981(0.973-0.989)$ & $0.969(0.947-0.990)$ & $0.993(0.983-1.000)$ & $0.977(0.958-0.996)$ \\
\hline & 3 year & $0.974(0.965-0.983)$ & $0.962(0.937-0.987)$ & $0.991(0.979-1.000)$ & $0.972(0.949-0.995)$ \\
\hline
\end{tabular}

Abbreviations: KM, Kaplan Meier; TAVI, Transcatheter Aortic Valve Implantation; SAVR, Surgical Aortic Valve Replacement; MI, Myocardial Infarction

eTable 3 Unadjusted and weighted Cox analysis (TAVI vs. SAVR estimates at 3-years follow-up).

\begin{tabular}{lcccccc}
\hline $\begin{array}{l}\text { Outcome of } \\
\text { interest }\end{array}$ & $\begin{array}{c}\text { Unadjusted } \\
\text { HR }\end{array}$ & $\begin{array}{c}\text { Unadjusted } \\
\mathbf{9 5 \%} \text { CI }\end{array}$ & p-value & $\begin{array}{c}\text { Adjusted } \\
\text { HR }\end{array}$ & $\begin{array}{c}\text { Weighted } \\
\mathbf{9 5 \%} \text { CI }\end{array}$ & p-value \\
\hline Death & 2.234 & $1.772-2.816$ & $<0.001$ & 1.334 & $0.955-1.863$ & 0.091 \\
Stroke & 1.187 & $0.710-1.985$ & 0.513 & 1.028 & $0.506-2.089$ & 0.940 \\
MI & 1.803 & $1.108-2.933$ & 0.018 & 1.432 & $0.686-2.986$ & 0.339 \\
Revascularization & 1.399 & $0.671-2.918$ & 0.371 & 2.860 & $0.744-11.001$ & 0.126 \\
\hline
\end{tabular}

Abbreviations: TAVI, Transcatheter Aortic Valve Implantation; SAVR, Surgical Aortic Valve Replacement; MI, myocardial infarction. 


\section{eFIGURES}

eFigure 1 Propensity score balance (Panel A showing absolute standardized differences before and after weighting (closed circles represent variables with statistically significant difference), Panel B showing quantiles of $\mathrm{p}$-values before and after weighting).
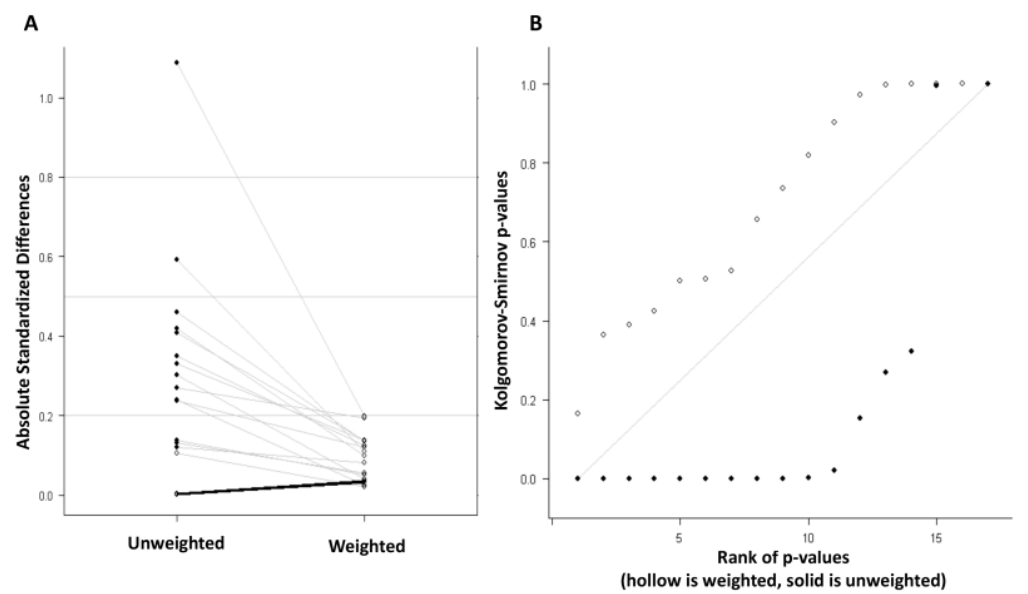

\section{eFigure 2.}

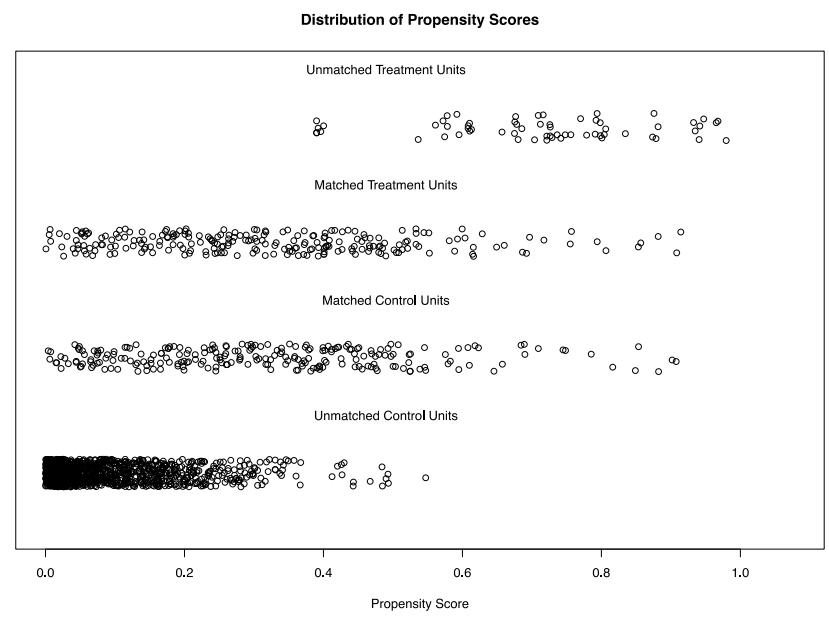


eFigure 3 Cox-predicted time-to-event curves for the principal outcomes of interest.
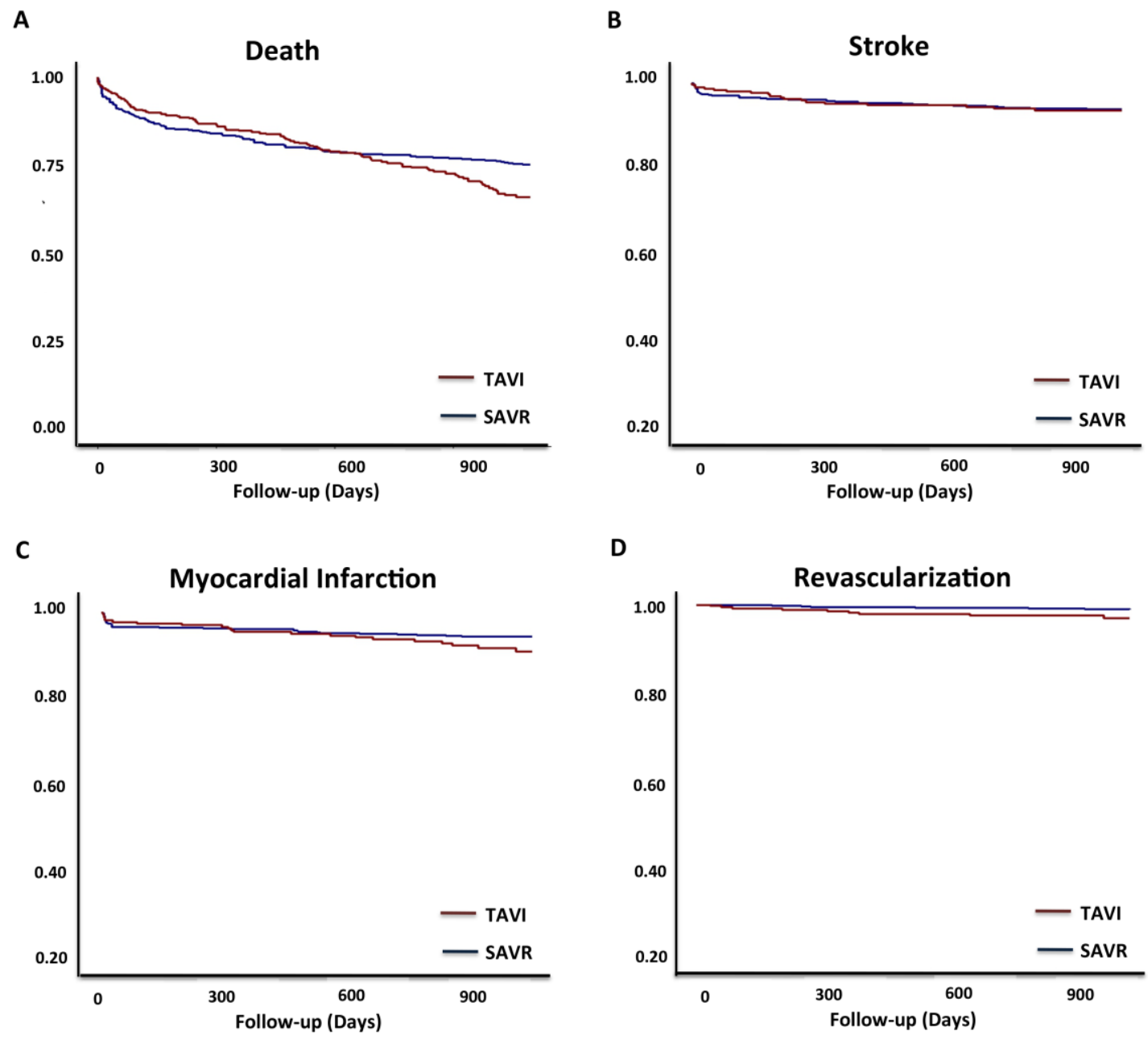

\section{OBSERVANT Research Group,}

Fulvia Seccareccia, Paola D’Errigo, Stefano Rosato, Alice Maraschini, Gabriella Badoni,

National Centre for Epidemiology, Surveillance and Health Promotion - ISS; Corrado Tamburino, Marco Barbanti, SICI-GISE, Gennaro Santoro, FIC, ANMCO; Francesco Santini, Francesco Onorati, Claudio Grossi, SICCH; Marco Ranucci, Remo Daniel Covello, ITACTA; Danilo Fusco, Epidemiology Dept. Lazio Region; Rossana De Palma, Emilia Romagna Region; Salvatore Scondotto, Sicilia Region.

\section{Participating hemodynamic centers}

1. Città della Salute e della Scienza - A.O.U. Molinette - San Giovanni Battista di Torino, Torino. Marra S., Marra S., D'Amico M. 
2. Città della Salute e della Scienza - A.O.U. Molinette - San Giovanni Battista di Torino, Torino. Gaita F., Moretti C.

3. Ospedale Mauriziano "Umberto I", Torino. De Benedictis M., Aranzulla T.

4. A.O. Nazionale Ss. Antonio e Biagio e Cesare Arrigo, Alessandria. Pistis G., Reale M.

5. Istituto Clinico S.Ambrogio, Milano. Bedogni F., Brambilla N.

6. Fondazione IRCSS Policlinico San Matteo, Pavia. Ferrario M., Ferrero L., Vicinelli P.

7. Fondazione San Raffaele del Monte Tabor, Milano. Colombo A., Chieffo A., Ferrari A.

8. I.R.C.C.S. Policlinico San Donato, San Donato M.se (MI). Inglese L., Casilli F.

9. Spedali Civili di Brescia - Università, Brescia. Ettori F., Frontini M.

10. Ospedale Luigi Sacco - A.O. - Polo Universitario, Milano. Antona C., Piccaluga E.

11. A.O. Ospedale Niguarda Cà Granda, Milano. Klugmann S., De Marco F.

12. A.O. Bolognini Seriate, Seriate (BG). Tespili M., Saino A.

13. Fondazione Poliambulanza Istituto Ospedaliero, Brescia. Leonzi Ornella, Rizzi Andrea

14. Ospedale "S. Maria di Ca' Foncello", Treviso. Franceschini Grisolia E., Franceschini Grisolia E.

15. A.O. di Padova, Padova. Isabella G., Fraccaro C.

16. A.O.U. Santa Maria Della Misericordia di Udine, Udine. Proclemer A., Bisceglia T., Armellini I.

17. A.O.U. San Martino, Genova. Vischi M., Parodi E.

18. A.O.U. di Parma, Parma. Vignali L., Ardissimo D.

19. Policlinico S Orsola Malpighi, Bologna. Marzocchi A., Marrozzini C.

20. Maria Cecilia Hospital, Ravenna. Cremonesi A., Colombo F.

21. A.O.U. Pisana, Pisa. Petronio S., Giannini C.

22. A.O.U. Senese Le Scotte, Siena. Pierli C., Iadanza A.

23. A.O.U. Careggi, Firenze. Santoro G., Meucci F. 
24. Ospedale del Cuore Fondazione CNR Regione Toscana G. Monasterio, Massa. Berti S., Mariani M.

25. European Hospital, Roma. Tomai F., Ghini A.

26. A.O. S Camillo-Forlanini, Roma. Violini R., Confessore P.

27. Policlinico Gemelli Cardiologia, Roma. Crea F., Giubilato S.

28. Policlinico Umberto I, Roma. Sardella G., Mancone M.

29. A.O.U. Integrata Verona, Verona. Ribichini F., Vassanelli C., Dandale R.

30. A.O.OO.RR.S. Giovanni di Dio e Ruggi d’Aragona - A.O.U. di Salerno, Salerno. Giudice P., Vigorito F.

31. Casa Di Cura Città Di Lecce Srl, Lecce. Liso A., Specchia L.

32. A.O.U. Mater Domini, Catanzaro. Indolfi C., Spaccarotella C.

33. A.R.N.A.S. Ospedale Civico - Di Cristina - Benfratelli, Palermo. Stabile A., Gandolfo C.

34. A.O.U. "Policlinico - Vittorio Emanuele" - Ospedale Ferrarotto, Catania. Tamburino C., Ussia G.

\section{Participating cardiac surgery centers}

1. Villa Maria Pia Hospital Gruppo Villa Maria Cardiochirurgia, Torino. Comoglio C., Dyrda O.

2. Città della Salute e della Scienza - A.O.U. Molinette - San Giovanni Battista di Torino, Torino. Rinaldi M., Salizzoni S.

3. Azienda Ospedaliera Universitaria Maggiore della Carità, Novara. Micalizzi E.

4. A.O. S. Croce e Carle, Cuneo. Grossi C., Di Gregorio O.

5. A.O. Nazionale Ss. Antonio e Biagio e Cesare Arrigo, Alessandria. Scoti P., Costa R.

6. Ospedale Mauriziano "Umberto I", Torino. Casabona R., Del Ponte S.

7. Istituto Clinico S.Ambrogio, Milano. Panisi P., Spira G.

8. Fondazione Poliambulanza Istituto Ospedaliero, Brescia. Troise G., Messina A.

9. Fondazione I.R.C.S.S. Policlinico San Matteo, Pavia. Viganò M., Aiello M. 
10. Fondazione San Raffaele del Monte Tabor, Milano. Alfieri O., Denti P.

11. I.R.C.C.S. Policlinico San Donato, San Donato M.se (MI). Menicanti L., Agnelli B.

12. IRCCS Multimedica, Milano. Donatelli F.

13. Spedali Civili di Brescia - Università, Brescia. Muneretto C., Frontini M.

14. Spedali Civili di Brescia - Università, Brescia. Rambaldini M., Frontini M.

15. A.O. Ospedale di Lecco - Presidio Alessandro Manzoni, Lecco. Gamba A., Tasca G.

16. Ospedali Riuniti di Bergamo - A.O., Bergamo. Ferrazzi P., Terzi A.

17. Ospedale Luigi Sacco - A.O. - Polo Universitario, Milano. Antona C., Gelpi G.

18. A.O. Ospedale Niguarda Cà Granda, Milano. Martinelli L., Bruschi G.

19. Presidio Ospedaliero S.Chiara - Ospedale di Trento, Trento. Graffigna A.C.

20. A.O.U. Integrata Verona, Verona. Mazzucco A.

21. A.O.U. Ospedali Riuniti di Trieste - Ospedale di Cattinara, Trieste. Pappalardo A., Gatti G.

22. A.O.U. Santa Maria Della Misericordia di Udine, Udine. Livi U., Pompei E.

23. ICLAS - Istituto Clinico Ligure di Alta Specialità , Rapallo (GE). Coppola R., Gucciardo M.

24. A.O.U. San Martino, Genova. Passerone G., Parodi E.

25. Salus Hospital spa, Reggio Emilia. Albertini A., Caprili L.

26. Hesperia Hospital Modena S.r.l., Modena. Ghidoni I., Gabbieri D.

27. Maria Cecilia Hospital, Ravenna. La Marra M., Aquino T.

28. Azienda Ospedaliero - Universitaria di Parma, Parma. Gherli T.

29. Policlinico S. Orsola Malpighi, Bologna. Di Bartolomeo R., Savini C.

30. Villa Maria Beatrice Hospital, Firenze. Popoff G., Innocenti D.

31. A.O.U. Pisana, Pisa. Bortolotti U., Pratali S.

32. A.O.U. Careggi, Firenze. Stefano P., Blanzola C.

33. Ospedale del Cuore Fondazione CNR Regione Toscana G. Monasterio, Massa. Glauber M., Cerillo A., Chiaramonti F.

34. A.O. Santa Maria, Terni. Pardini A., Fioriello F. 
35. A.O. G. M. Lancisi, Ancona. Torracca L., Rescigno G.

36. European Hospital, Roma. De Paulis R., Nardella S.

37. A.O. S Camillo-Forlanini, Roma. Musumeci F., Luzi G.

38. Policlinico Gemelli, Roma. Possati G., Bonalumi G.

39. Università Campus Bio-Medico di Roma, Roma. Covino E., Pollari F.

40. A.O. Sant'Andrea, Roma. Sinatra R., Roscitano A.

41. Policlinico Tor Vergata, Roma. Chiariello L., Nardi P.

42. Clinica San Michele, Maddaloni (CS). Lonobile T., Baldascino F.

43. A.O.OO.RR.S. Giovanni di Dio e Ruggi d`Aragona - A.O.U. di Salerno, Salerno. Di Benedetto G., Mastrogiovanni G.

44. A.O. San Sebastiano, Caserta. Piazza L., Marmo J.

45. A.O.U. Federico II, Napoli. Vosa C., De Amicis V.

46. Anthea Hospital, Bari. Speziale G., Visicchio G., Spirito R.

47. Casa Di Cura Citta Di Lecce Srl, Lecce. Gregorini R., Specchia L.

48. Azienda Sanitaria Locale Le Fazzi Presidio Ospedaliero Vito Fazzi, Lecce. Villani M., Pano M.A.

49. A.O.U. Consorziale Policlinico di Bari, Bari. Bortone A., De Luca Tupputi Schinosa L., De Cillis E.

50. Azienda Ospedaliera Regionale San Carlo, Potenza. Gaeta R., Di Natale M.

51. S. Anna Hospital, Catanzaro. Cassese M., Antonazzo A.

52. Villa Maria Eleonora Hospital, Palermo. Argano V., Santaniello E.

53. Centro Cuore Morgagni, Pedara (CT). Patanè L., Gentile M., Tribastone S.

54. A.R.N.A.S. Ospedale Civico - Di Cristina - Benfratelli, Palermo. Follis F., Montalbano G.

55. IS.ME.T.T. (Istituto Mediterraneo per i Trapianti e Terapie ad Alta Specializzazione), Palermo. Pilato M., Stringi V.

56. A.O. Ospedali Riuniti Papardo - Piemonte, Messina. Patanè F., Salamone G. 
57. A.O.U. Policlinico Paolo Giaccone, Palermo. Ruvolo G., Pisano C.

58. A.O.U. "Policlinico - Vittorio Emanuele" - Ospedale Ferrarotto, Catania. Mignosa C., Bivona A.

59. A.O. Brotzu, Cagliari. Cirio E.M., Lixi G. 